Diabetologe 2020 · 16:433-434

https://doi.org/10.1007/s11428-020-00635-6

(c) Springer Medizin Verlag $\mathrm{GmbH}$, ein Teil von Springer Nature 2020

Das 15. Diabetes Update fand am 22. und 23.03.2020 statt. Es war ein Update der anderen Art: Alle Vorträge wurden zwar wie geplant gehalten, sie wurden aber, bedingt durch die Coronapandemie, als Stream-Update verfügbar gemacht. Dabei hatten alle Teilnehmerinnen und Teilnehmer wie immer die Möglichkeit, nach den einzelnen Blöcken und am Ende jedes Vortrags online Fragen zu stellen, die auch direkt beantwortet wurden. In diesem Kontext zeigte sich auch die neue Realität; eine Kollegin verfolgte z.B. die Vorträge am PC („personal computer"), während sie gleichzeitig die Kinder um sich herum beschäftigen musste, die wegen der Schließung von Kindergärten und Schulen zu Hause waren. Dank der exzellenten Vorbereitungen der Organisatoren von med update lief alles technisch auf höchstem Niveau, und die Moderatoren aus dem Kreis der wissenschaftlichen Leitung von Diabetes Update verstanden es exzellent $\mathrm{zu}$ vermitteln, dass es sich um eine Liveveranstaltung handelt, bei der es sich lohnt, mit dabei zu sein.

\section{》) Aufgrund der Corona- epidemie wurde das 15. Diabe- tes Update als Stream-Update verfügbar gemacht}

Wie in den Jahren zuvor werden auch dieses Mal einige ausgewählte Themen im Diabetes-Update-Heft von Der Diabetologe publiziert. Die wichtigsten Botschaften und neuen Erkenntnisse aus den anderen Vorträgen werden in dem Beitrag „Diabetes Update 2020: Das Wichtigste für die ärztliche Praxis" in diesem Heft vom Unterzeichner zusammengestellt.

Werner A. Scherbaum

Universitätsklinikum Düsseldorf, Heinrich-Heine-Universität Düsseldorf, Düsseldorf, Deutschland

\title{
Ein Diabetes-Update der anderen Art
}

\section{Typ-2-Diabetes}

Die in diesem Heft ausführlich dargestellten Beiträge betreffen zum einen den Typ2-Diabetes, ein Kernthema unseres Fachgebiets, vorgetragen durch Herrn Professor Andreas Hamann, Chefarzt an den Hochtaunus-Kliniken in Bad Homburg. Diesen Beitrag möchte ich Ihnen ganz besonders ans Herz legen, weil der Autor die aktuellen Änderungen im therapeutischen Vorgehen beim Typ-2-Diabetes sehr klar aufzeigt und diese auch durch die neue wissenschaftliche Evidenz gut begründet.

\section{Diabetes und Herz}

Dieses Thema ist aus der Diabetologie und auch der Kardiologie und der Allgemeinmedizin nicht mehr wegzudenken. Professor Michael Lehrke von der Klinik für Kardiologie, Angiologie und Internistische Intensivmedizin des Universitätsklinikums Aachen zeigt in seinem Beitrag sehr überzeugend auf, welch wichtige Rolle inzwischen die Therapie mit SGLT2-Hemmern (SGLT-2: „sodium dependent glucose transporter 2“) und GLP-1Rezeptor-Agonisten (GLP-1: „glucagonlike peptide $1^{\text {") }}$ bei kardiologischen $\mathrm{Pa}$ tientinnen und Patienten mit Diabetes einnimmt. Für besonders beachtenswert halte ich auch seine Ausführungen zu den neuen ESC-Leitlinien (ESC: „European Society of Cardiology“) zur Lipidtherapie.

\section{Diabetes und Schwangerschaft}

Brillant war einmal wieder der Vortrag von Dr. Helmut Kleinwechter aus Kiel, und nicht weniger klar und lesenswert ist auch sein Beitrag zum Thema Diabetes und Schwangerschaft in diesem Heft. Beim Diabetes Update hatte er auch über den ersten deutschen Fall einer COVID-19-Erkrankung bei Gestationsdiabetes berichtet, der aus Gründen der Aktualität bereits separat als ausführliche Fallbeschreibung im Mai-Heft von Der Diabetologe publiziert worden ist und daher hier nur kurz aufgezeigt wird [1]. Inzwischen hat die Deutsche Gesellschaft für Neonatologie (DGPM) das wohl einzigartige Register CRONOS („COVID19 related obstetrics and national outcome study in Germany") aufgelegt, bei dem alle Schwangeren mit einer SARS-CoV-2-Infektion („severe acute respiratory syndrome coronavirus 2“) erfasst und ggf. beraten werden. Dabei ist Herr Dr. Kleinwechter zusammen mit Frau Dr. Katharina Laubner, Oberärztin an der Abteilung Endokrinologie und Diabetologie des Universitätsklinikums Freiburg, für die Aufarbeitung der Diabetesfälle zuständig. Hiermit möchte ich auch von dieser Stelle aus alle Kolleginnen und Kollegen, die Schwangere mit Diabetes betreuen, sehr dazu ermuntern, sich an diesem wertvollen Register zu beteiligen.

\section{Impfempfehlungen}

Schließlich und nicht zuletzt möchte ich Sie auf die Lektüre des Hot-TopicsBeitrags „Impfungen“ hinweisen, der auch in Bezug zur COVID-19-Pandemie von ganz besonderer Aktualität ist. Herr Professor Fred Zepp, Direktor des Zentrums für Kinder- und Jugendmedizin, Universitätsmedizin Mainz, ist seit vielen Jahren spezialisiert auf Impfstoffentwicklung und -forschung, Mitglied der Ständigen Impfkommission (STIKO) des Robert Koch-Instituts und (einziges) 
deutsches Mitglied des Wissenschaftlichen Beirats des „International Vaccine Institute" (IVI) der Vereinten Nationen. Aus erster Hand berichtet er in seinem Artikel über allgemeine Impfempfehlungen und geht speziell auf Empfehlungen für Diabetespatientinnen und -patienten in verschiedenen Altersgruppen ein. Die Erkenntnis, dass Menschen mit Diabetes ganz besonders von den angebotenen Impfungen profitieren, sollte unsere Kolleginnen und Kollegen dazu ermuntern, bei den von ihnen betreuten Patientinnen und Patienten regelmäßig den Impfstatus zu überprüfen.

Ich wünsche Ihnen viel Spaß und viele wichtige Anregungen beim Lesen dieses Update-Hefts!

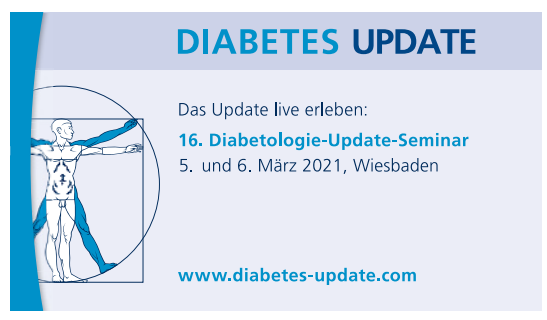

Ihr

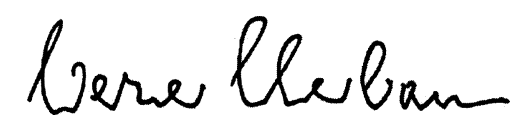

Werner Scherbaum

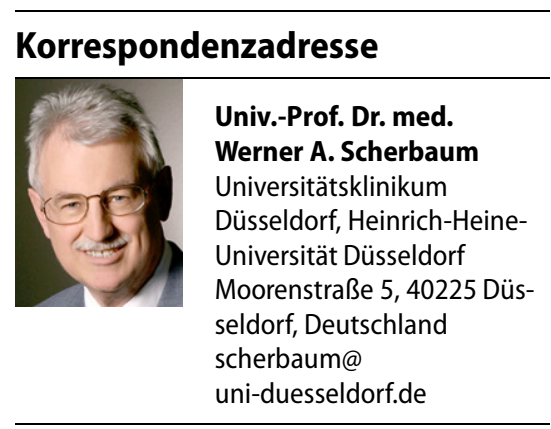

Interessenkonflikt. W.A. Scherbaum gibt an, dass kein Interessenkonflikt besteht.

\section{Literatur}

1. Kleinwechter H, Laubner K (2020) Coronaviruserkrankung 2019 (COVID-19) und Schwangerschaft. Diabetologe 16:242-246. https://doi.org/10. 1007/s11428-020-00611-0

\section{Transition: Jugendliche mit Diabetes Typ 1 vor besonderen Herausforderungen}

\section{Wenn in der Pubertät der Blutzucker verrücktspielt}

Kinder mit Diabetes Typ 1 und ihre Familien lernen zunächst meist schnell, mit der Stoffwechselerkrankung und der damit verbundenen Therapie zu leben. Doch etwa ab einem Alter von 10 Jahren setzen die körperlichen Veränderungen der Pubertät ein: „Plötzlich werden vermehrt und ungleichmäßig Sexualhormone ausgeschüttet. Sie senken die Insulinempfindlichkeit und verursachen Blutzuckerschwankungen. Außerdem setzt der Körper verstärkt Wachstumshormone frei, die zu hohen morgendlichen Blutzuckerwerten führen können, auch bekannt als Dawn-Phänomen", erklärt die Diabetesberaterin Andrea Witt. Dadurch kann der Langzeitblutzuckerwert $\mathrm{HbA1C}$ deutlich über die empfohlene Spanne von 6,5 bis 7 Prozent (48 bis $53 \mathrm{mmol} / \mathrm{mol}$ ) ansteigen. Das führt bei den jungen Betroffenen zu Frust - wozu sich bei der Therapie Mühe geben, wenn die Werte sowieso „Achterbahn fahren"?

Darüber hinaus möchten viele Jugendliche ihren Alltag flexibel gestalten, ohne jedes Mal die Insulindosis beim Sport oder Essen anzupassen. „Daher passiert es schon mal öfter, dass sie nicht an die nötige Insulingabe denken“, weiß Andrea Witt. Hinzu kommt: Viele Jugendliche machen erste Erfahrungen in der Liebe, mit Alkohol oder Drogen, sie plagen sich mit Selbstzweifeln und Konflikten in Schule oder Elternhaus. Vorwürfe rund um die Diabesttherapie fördern in dieser Situation zusätzlich die Ausschüttung von Stresshormonen, was wiederum den Blutzuckerspiegel weiter in die Höhe treibt. In dieser Phase ist deshalb eine regelmäßige Betreuung und Beratung besonders wichtig, betont Andrea Witt: „Jugendliche mit Diabetes Typ 1 benötigen umfassende Aufklärung über ihre Erkrankung, zum Beispiel zur Gefahr einer Unterzuckerung bei Alkoholkonsum oder beim Sex."

Der nächste bedeutende Lebensabschnitt ist die Transition, der Übergang vom Kinderdiabetologen in eine Schwerpunktpraxis für Erwachsene. Auch diese Veränderung kann herausfordernd sein, sei es die Suche nach einem passenden Arzt, die nun generell höhere Eigenverantwortung oder die im Vergleich zum Kinderdiabetologen weniger engmaschige Betreuung und Fürsorge. So mancher junger Erwachsene geht daher mitunter jahrelang gar nicht mehr zum Diabetologen. Die Konsequenz können in späteren Jahren auftretende Folgeerkrankungen und Komplikationen sein.

Tipps für Jugendliche, Eltern und Betreuer, Stress in der Pubertät zu vermeiden und die Diabetes-Therapie nicht zu vernachlässigen, gibt die diabetesDE-SorgentelefonExpertin einmal monatlich jeweils donnerstags von 18 bis 20 Uhr. Die aktuellen Termine finden Interessierte im Internet unter: https://www.diabetesde.org/sorgentelefon-eltern-kindern-diabetes-typ-1

Quelle: www.diabetesde.org 